\title{
On a Quarter-Symmetric Projective Conformal Connection
}

\author{
Wanxiao Tang, Tal Yun Ho, Fengyun Fu and Peibiao Zhao* \\ (Communicated by Uday Chand De)
}

\begin{abstract}
We introduce a class of quarter-symmetric projective conformal connections, and study the geometrical properties of a manifold associated with this connection. The Schur's theorem corresponding to the quarter-symmetric projective conformal connection is derived.
\end{abstract}

Keywords: quarter-symmetric projective conformal connection; conjugate symmetry; constant curvature.

AMS Subject Classification (2010): Primary:53C20; Secondary: 53D11.

\section{Introduction}

Since A. Fridman and J. A. Schouten [6] first introduced the concept of the semi-symmetric linear connection, afterwards, using [10], the metric connection with a torsion was deeply studied. K. Yano in [12] defined a semisymmetric metric connection and studied its geometric properties. In [18], a semi-symmetric connection that is projectively equivalent to the Levi-Civita connection was called a projective semi-symmetric connection and its properties were considered. In $[15,19,20,2,5,8,9]$, these connections were more deeply studied. In [16], a mutual connection and its dual connection of the semi-symmetric metric connection were considered. And in [11] a conjugate symmetry condition of the Amari-Chentsov connection was considered. In [14], one type of semi-symmetric non-metric connections satisfying the Schur's theorem was investigated. K. Yano and J. Imai [13] defined and studied a quarter-symmetric metric connection generalizing semi-symmetric metric connection. U. C. De and S. C. Biswas [1] studied quarter-symmetric metric connection in a SP-Sasakian manifold. Han, Ho and Zhao [7] obtained a projective invariant of quarter-symmetric metric connections. In $[4,20,3]$ the projective property of the quarter-symmetric metric connection was studied. In [17] semisymmetric projective conformal connection was newly defined and the semi-symmetric projective conformal connection satisfying the Schur's theorem was studied.

In this paper, we newly define, motivated by $[1,4,7]$, the quarter-symmetric projective conformal connection and study its properties. And the quarter-symmetric projective conformal connection satisfying the Schur's theorem is studied.

\section{Main Results}

On a Riemannian manifold $(M, g)$, quarter-symmetric metric connection $\stackrel{q}{\nabla}$ satisfies the relation

$$
\left(\stackrel{q}{\nabla}_{z} g\right)(X, Y)=0, \quad \stackrel{q}{T}(X, Y)=\varphi(X) \pi(Y)-\varphi(Y) \pi(X) .
$$

where $\varphi$ is $(1,1)$-type tensor field and $\pi$ is a 1 -form.

Local expression of this expression is

$$
\stackrel{q}{\nabla_{k}} g_{i j}=0, \quad \stackrel{q}{T_{i j}^{k}}=\pi_{j} \varphi_{i}^{k}-\pi_{i} \varphi_{j}^{k},
$$

Received : 06-09-2016, Accepted : 03-03-2017

* Corresponding author

This research supported by a Grant-in-Aid for NNSF of China (11371194) and by a Grant-in-Aid for Jiangsu excellent graduate course"Portfolio management+micro course" and by NUST Research Funding No. CXZZ11-0258. 
and the connection coefficient is

$$
\stackrel{q}{\Gamma_{i j}^{k}}=\left\{\begin{array}{l}
k \\
i j
\end{array}\right\}+\pi_{j} U_{i}^{k}-\pi_{i} V_{j}^{k}-U_{i j} \pi^{k},
$$

where $\left\{{ }_{i j}^{k}\right\}$ is the coefficient of the Levi-Civita connection and $\varphi_{i}^{j}$ and $\pi_{i}$ are components of $(1,1)$-type tensor field and 1-form $\pi$ respectively and $U_{i j}=\frac{1}{2}\left(\varphi_{i j}+\varphi_{j i}\right), \quad V_{i j}=\frac{1}{2}\left(\varphi_{i j}-\varphi_{j i}\right)$ and $\pi^{k}=g^{k l} \pi_{l}([7])$.

Definition 2.1. In a Riemannian manifold, connection $\stackrel{p}{\nabla}$ is called a quarter-symmetric projective connection, if $\stackrel{p}{\nabla}$ is projectively equivalent to a quarter-symmetric metric connection $\stackrel{q}{\nabla}$.

In a Riemannian manifold, a quarter-symmetric projective connection $\stackrel{p}{\nabla}$ satisfies the relation

$$
\begin{gathered}
\left(\stackrel{p}{\nabla}_{z} g\right)(X, Y)=-2 \psi(Z) g(X, Y)-\psi(X) g(Y, Z)-\psi(Y) g(X, Z), \\
\stackrel{p}{T}(X, Y)=\varphi(X) \pi(Y)-\varphi(Y) \pi(X),
\end{gathered}
$$

and the coefficient of $\stackrel{p}{\nabla}$ is

$$
\stackrel{p}{\Gamma_{i j}^{k}}=\left\{\begin{array}{l}
k \\
i j
\end{array}\right\}+\psi_{i} \delta_{j}^{k}+\psi_{j} \delta_{i}^{k}+\pi_{j} U_{i}^{k}-\pi_{i} U_{j}^{k}-U_{i j} \pi^{k}
$$

Definition 2.2. In a Riemannian manifold, connection $\nabla^{c}$ is called a quarter-symmetric conformal connection, if $\stackrel{c}{\nabla}$ is conformally equivalent to a quarter-symmetric metric connection $\stackrel{q}{\nabla}$.

In a Riemannian manifold, a quarter-symmetric conformal connection $\stackrel{c}{\nabla}$ satisfies the relation

$$
\left(\stackrel{c}{\nabla}_{z} \bar{g}\right)(X, Y)=2 Z \sigma \bar{g}(X, Y), \quad \stackrel{c}{T}(X, Y)=\varphi(X) \pi(Y)-\varphi(Y) \pi(X),
$$

where

$$
\bar{g}(X, Y)=e^{2 \sigma(x)} g(X, Y)
$$

And the coefficient of $\stackrel{c}{\nabla}$ is

$$
\stackrel{c}{\Gamma_{i j}^{k}}=\overline{\left\{{ }_{i j}^{k}\right\}}-\sigma_{i} \delta_{j}^{k}-\sigma_{j} \delta_{i}^{k}+\bar{g}_{i j} \sigma^{k}+\pi_{j} U_{i}^{k}-\pi_{i} V_{j}^{k}-U_{i j} \pi^{k} .
$$

where $\left.\overline{\{i j}{ }_{i j}^{k}\right\}$ is the coefficient of the Levi-Civita connection $\stackrel{0}{\nabla}$ of conformal metric $\bar{g}_{i j}=e^{2 \sigma(x)} g_{i j}$ and $\sigma_{i}=\partial_{i} \sigma$.

Definition 2.3. In a Riemannian manifold, connection $\nabla$ is called a quarter-projective conformal connection, if $\nabla$ is projective and conformal equivalent to a quarter-symmetric metric connection $\stackrel{q}{\nabla}$.

In a Riemannian manifold, a quarter-symmetric projective conformal connection $\nabla$ satisfies the relation

$$
\begin{gathered}
\nabla_{z} \bar{g}(X, Y)=-2[\psi(Z)-Z \sigma] \bar{g}(X, Y)-\psi(X) \bar{g}(Y, Z)-\psi(Y) \bar{g}(X, Z), \\
T(X, Y)=\varphi(X) \pi(Y)-\varphi(Y) \pi(X) .
\end{gathered}
$$

The local expression of the relation (2.4) is

$$
\nabla_{k} \bar{g}_{i j}=-2\left(\psi_{k}-\sigma_{k}\right) \bar{g}_{i j}-\psi_{i} \bar{g}_{j k}-\psi_{j} \bar{g}_{i k}, \quad T_{i j}^{k}=\pi_{j} \varphi_{i}^{k}-\pi_{i} \varphi_{j}^{k}
$$

and its coefficient is

$$
\Gamma_{i j}^{k}=\overline{\left\{{ }_{i j}^{k}\right\}}+\left(\psi_{i}-\sigma_{i}\right) \delta_{j}^{k}+\left(\psi_{j}-\sigma_{j}\right) \delta_{i}^{k}+g_{i j} \sigma^{k}+\pi_{j} U_{i}^{k}-\pi_{i} V_{j}^{k}-U_{i j} \pi^{k} .
$$

Remark 2.1. If $\sigma=0$, then the quarter-symmetric projective conformal connection $\nabla$ is $\nabla=\stackrel{p}{\nabla}$; If $\psi=0$, then the quarter-symmetric projective conformal connection $\nabla$ is $\nabla=\stackrel{c}{\nabla}$; If $\sigma=\psi=0$, then the quartersymmetric projective conformal connection $\nabla$ is $\nabla=\stackrel{q}{\nabla}$. And if $\varphi(X)=X$, then the quarter-symmetric projective conformal connection $\nabla$ is a semi-symmetric projective conformal connection[17]. 
From (2.6), we find that the curvature tensor of $\nabla$ is

$$
\begin{aligned}
R_{i j k}^{l}= & \bar{K}_{i j k}^{l}+\delta_{j}^{l} a_{i k}-\delta_{i}^{l} a_{j k}+b_{i}^{l} \bar{g}_{j k}-b_{j}^{l} \bar{g}_{i k}+U_{j}^{l} c_{i k}-U_{i}^{l} c_{j k} \\
& +c_{j}^{l} U_{i k}-c_{i}^{l} U_{j k}+U_{i j}^{l} \pi_{k}-U_{i j k} \pi^{l}-V_{k}^{l} \pi_{i j}+V_{j k}^{l} \pi_{i} \\
& -V_{i k}^{l} \pi_{j}+\delta_{k}^{l} \psi_{i j}+T_{i j}^{l} \psi_{k}+\delta_{j}^{l} V_{k}^{p} \psi_{p} \pi_{i}-\delta_{i}^{l} V_{k}^{p} \psi_{p} \pi_{j},
\end{aligned}
$$

where $\bar{K}_{i j k}^{l}$ is the curvature tensor of $\stackrel{0}{\nabla}$ of $\bar{g}_{i j}$,

$$
\begin{aligned}
& a_{i k}=\stackrel{0}{\nabla}_{i}\left(\psi_{k}-\sigma_{k}\right)-\left(\psi_{i}-\sigma_{i}\right)\left(\psi_{k}-\sigma_{k}\right)-U_{i}^{p}\left(\psi_{p}-\sigma_{p}\right) \pi_{k}-\bar{g}_{i k}\left(\psi_{p}-\sigma_{p}\right) \sigma^{p}+U_{i k}\left(\psi_{p}-\sigma_{p}\right) \pi^{p}, \\
& b_{i k}=\stackrel{0}{\nabla}_{i} \sigma_{k}+\sigma_{i} \sigma_{k}-U_{i p} \sigma^{p} \pi_{k}+U_{i k} \sigma^{p} \pi_{p}, \\
& c_{i k}=\stackrel{0}{\nabla}_{i} \pi_{k}+\pi_{i} \pi_{k}-U_{i p} \pi^{p} \pi_{k}+\frac{1}{2} U_{i k} \pi^{p} \pi_{p}, \\
& U_{i j}^{l}=\stackrel{0}{\nabla}_{i} U_{j}^{l}-\stackrel{0}{\nabla}_{j} U_{i}^{l} \\
& U_{i j k}=U_{i j}^{l} g_{l k} \\
& V_{i k}^{l}=\stackrel{0}{\nabla}_{i} V_{k}^{l}-U_{i}^{p} V_{p}^{l} \pi_{k}+U_{i}^{l} V_{k}^{p} \pi_{p}+U_{i k} V_{p}^{l} \pi^{p}-U_{i p} V_{k}^{p} \pi^{l}+V_{i}^{l} \sigma_{k}-V_{i k} \sigma^{l}-\delta_{i}^{l} V_{k}^{p} \sigma_{p}-\bar{g}_{i k} V_{p}^{l} \sigma^{p}, \\
& \psi_{i j}=\stackrel{0}{\nabla}_{i} \psi_{j}-\stackrel{0}{\nabla}_{j} \psi_{i}, \\
& \pi_{i j}=\stackrel{0}{\nabla}_{i} \pi_{j}-\stackrel{0}{\nabla}_{j} \pi_{i} .
\end{aligned}
$$

From (2.5), dual connection $\stackrel{*}{\nabla}$ of the quarter-symmetric projective conformal connection $\nabla$ satisfies the relation

$$
\stackrel{*}{\nabla}_{k} \bar{g}_{i j}=2\left(\psi_{k}-\sigma_{k}\right) \bar{g}_{i j}+\psi_{i} \bar{g}_{j k}+\psi_{j} \bar{g}_{i k}, \stackrel{*}{T_{i j}^{k}}=\left(\pi_{j}-2 \sigma_{j}+\psi_{j}\right) \delta_{i}^{k}-\left(\pi_{i}-2 \sigma_{i}+\psi_{i}\right) \delta_{j}^{k},
$$

and its coefficient is

$$
\Gamma_{i j}^{*}=\left\{\begin{array}{l}
k \\
i j
\end{array}\right\}-\left(\psi_{i}-\sigma_{i}\right) \delta_{j}^{k}-\sigma_{j} \delta_{i}^{k}-\bar{g}_{i j}\left(\psi^{k}-\sigma^{k}\right)+\pi_{j} U_{i}^{k}-\pi_{i} V_{j}^{k}-U_{i j} \pi^{k} .
$$

And the curvature tensor is

$$
\begin{aligned}
R_{i j k}^{*}= & \bar{K}_{i j k}^{l}+\delta_{i}^{l} b_{j k}-\delta_{j}^{l} b_{i k}+a_{j}^{l} \bar{g}_{i k}-a_{i}^{l} \bar{g}_{j k}+U_{j}^{l} c_{i k}-U_{i}^{l} c_{j k} \\
& +c_{j}^{l} U_{i k}-c_{i}^{l} U_{j k}+U_{i j}^{l} \pi_{k}-U_{i j k} \pi^{l}-V_{k}^{l} \pi_{i j}+V_{j k}^{l} \pi_{i} \\
& -V_{i k}^{l} \pi_{j}-\delta_{k}^{l} \psi_{i j}-T_{i j k} \psi^{l}+\bar{g}_{j k} V_{p}^{l} \psi^{p} \pi_{i}-\bar{g}_{i k} V_{p}^{l} \psi^{p} \pi_{j} .
\end{aligned}
$$

From the expressions (2.7) and (2.9)

$$
\begin{aligned}
R_{i j k}^{*}= & R_{i j k}^{l}+\delta_{i}^{l} \alpha_{j k}-\delta_{j}^{l} \alpha_{i k}+\alpha_{j}^{l} \bar{g}_{i k}-\alpha_{i}^{l} \bar{g}_{j k}-2 \delta_{k}^{l} \psi_{i j}-T_{i j k} \psi^{l} \\
& -T_{i j}^{l} \psi_{k}+\bar{g}_{j k} V_{p}^{l} \psi^{p} \pi_{i}-\bar{g}_{i k} V_{p}^{l} \psi^{p} \pi_{j}+\delta_{i}^{l} V_{k}^{p} \psi_{p} \pi_{j}-\delta_{j}^{l} V_{k}^{p} \psi_{p} \pi_{i},
\end{aligned}
$$

where $\alpha_{j k}=a_{j k}+b_{j k}, T_{i j k}=T_{i j}^{l} g_{l k}$.

Theorem 2.1. In a Riemannian manifold, if a 1-form $\psi$ is a closed form, then a volume curvature tensor of the quartersymmetric projective conformal connection $\nabla$ is zero, namely

$$
P_{i j}=0
$$

where $P_{i j}$ is a volume curvature tensor of $\nabla$.

Proof. Contracting the indices $\mathrm{k}$ and 1 of (2.7), then we obtain

$$
\begin{aligned}
& P_{i j}=\stackrel{0}{P}_{i j}+a_{i j}-a_{j i}+b_{i j}-b_{j i}+U_{j}^{k} c_{i k}-U_{i}^{k} c_{j k}+U_{i k} c_{j}^{k}-U_{j k} c_{i}^{k}+U_{i j}^{k} \pi_{k}-U_{i j k} \pi^{k} \\
& -V_{k}^{k} \pi_{j}+V_{j k}^{k} \pi_{i}-V_{i k}^{k} \pi_{j}+n \psi_{i j}+T_{i j}^{k} \psi_{k}+V_{j}^{p} \psi_{p} \pi_{i}-V_{i}^{p} \psi_{p} \pi_{j},
\end{aligned}
$$


where $\stackrel{0}{P}_{i j}$ is a volume curvature tensor of the Levi-Civita connection $\frac{0}{\nabla}$ of $\bar{g}_{i j}, U_{i}^{j}=U_{i k} g^{k j}, V_{i}^{j}=V_{i k} g^{k j}$ and $c_{i}^{j}=c_{i k} g^{k j}$. That is to say

$$
\begin{aligned}
P_{i j}= & \bar{P}_{i j}+\left(a_{i j}-a_{j i}+b_{i j}-b_{j i}+V_{j}^{p} \psi_{p} \pi_{i}-V_{i}^{p} \psi_{p} \pi_{j}\right)+\left(U_{j}^{k} c_{i k}-U_{i}^{k} c_{j k}+U_{i k} c_{j}^{k}-U_{j k} c_{i}^{k}\right) \\
& +\left(U_{i j}^{k} \pi_{k}-U_{i j k} \pi^{k}\right)-V_{k}^{k} \pi_{j}+V_{j k}^{k} \pi_{i}-V_{i k}^{k} \pi_{j}+n \psi_{i j}+T_{i j}^{k} \psi_{k},
\end{aligned}
$$

On the other hand

$$
\begin{aligned}
& a_{i j}-a_{j i}+b_{i j}-b_{j i}+\left(V_{j}^{p} \pi_{i}-V_{i}^{p} \pi_{j}\right) \psi_{p}=\psi_{i j}-T_{i j}^{p} \psi_{p}, \\
& \bar{P}_{i j}=0, \quad U_{j}^{k} c_{i k}-U_{i}^{k} c_{j k}+U_{i k} c_{j}^{k}-U_{j k} c_{i}^{k}=0, \\
& U_{i j}^{k} \pi_{k}-U_{i j k} \pi^{k}=0, \quad V_{k}^{k}=0, \quad V_{j k}^{k}=0 .
\end{aligned}
$$

Hence

$$
P_{i j}=(n+1) \psi_{i j}
$$

If a 1 -form $\psi$ is a closed form, then $\psi_{i j}=0$. Hence from the expression (2.12), we obtain the expression (2.11).

Remark 2.2. Theorem 2.1 shows that the volume flat condition of the quarter-symmetric projective conformal connection $\nabla$ is independent of both quarter-symmetric component and conformal component, and depends only on projective component.

Theorem 2.2. The quarter-symmetric conformal connection on a Riemannian manifold $(M, g)$ is conjugate symmetric if and only if its Ricci curvature tensor is equal to that with respect to its dual connection.

Proof. From the expression (2.10), if $\psi=0$, then we obtain

$$
\stackrel{c *}{R_{i j k}^{l}}=\stackrel{c}{l} R_{i j k}^{l}+\delta_{i}^{l} \alpha_{j k}-\delta_{j}^{l} \alpha_{i k}+\bar{g}_{i k} \alpha_{j}^{l}-\bar{g}_{j k} \alpha_{i}^{l},
$$

Contracting the indices $i$ and 1 , then we obtain

$$
\stackrel{c *}{R_{j k}}=\stackrel{c}{R_{j k}}+n \alpha_{j k}-\bar{g}_{j k} \alpha_{i}^{i},
$$

From this expression, we find

$$
\alpha_{j k}=\frac{1}{n}\left(R_{j k}^{c *}-R_{j k}^{c}+\bar{g}_{j k} \alpha_{i}^{i}\right)
$$

Substituting this expression into the expression (2.13), we obtain

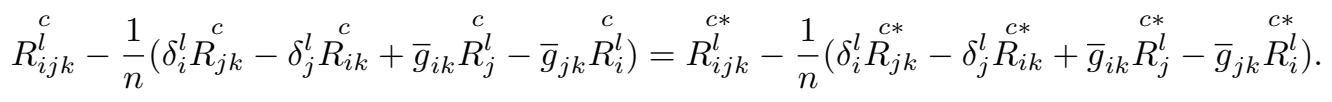

From this expression, there holds $R_{i j k}^{l *}=R_{i j k}^{l}$ if and only if $\stackrel{c *}{j k}_{j k}^{l}=\stackrel{c}{R_{j k}}$.

The second Bianchi identity of the curvature tensor $R_{i j k}^{l}$ of the quarter-symmetric projective conformal connection $\nabla$ on a Riemannian manifold $(M, g)$ is

$$
\nabla_{h} R_{i j k}^{l}+\nabla_{i} R_{j h k}^{l}+\nabla_{j} R_{h i k}^{l}=T_{h i}^{m} R_{j m k}^{l}+T_{i j}^{m} R_{h m k}^{l}+T_{j h}^{m} R_{i m k}^{l} .
$$

By $R_{i j k l}=g_{l p} R_{i j k}^{p}$, this expression becomes

$$
\nabla_{h} R_{i j k l}+\nabla_{i} R_{j h k l}+\nabla_{j} R_{h i k l}=\nabla_{h} g_{l p} R_{i j k}^{p}+\nabla_{i} g_{l p} R_{j h k}^{p}+\nabla_{j} g_{l p} R_{h i k}^{p}+T_{h i}^{m} R_{j m k l}+T_{i j}^{m} R_{h m k l}+T_{j h}^{m} R_{i m k l} .
$$

Theorem 2.3. Suppose a connected Riemannian manifold $(M, g)(\operatorname{dim} M \geq 3)$ associated with a quarter-symmetric projective conformal connection is everywhere isotropici. If

$$
\psi_{h}-2 \sigma_{h}+\frac{2}{n-1}\left(\pi_{h} \varphi_{p}^{p}-\pi_{p} \varphi_{h}^{p}\right)=0
$$

then $(M, g, \nabla)$ is a constant curvature manifold. 
Proof. If $(M, g, \nabla)$ is everywhere wandering, the curvature tensor is

$$
R_{i j k l}=K(p)\left(\bar{g}_{i l} \bar{g}_{j k}-\bar{g}_{i k} \bar{g}_{j l}\right),
$$

Substituting the expression (2.16) into (2.14) and using (2.5), then we obtain

$$
\begin{aligned}
& {\left[\nabla_{h} K-K\left(\psi_{h}-2 \sigma_{h}\right)\right]\left(\bar{g}_{i l} \bar{g}_{j k}-\bar{g}_{i k} \bar{g}_{j l}\right)+\left[\nabla_{i} K-K\left(\psi_{i}-2 \sigma_{i}\right)\right]\left(\bar{g}_{j l} \bar{g}_{h k}-\bar{g}_{j k} \bar{g}_{h l}\right)+} \\
& {\left[\nabla_{j} K-K\left(\psi_{j}-2 \sigma_{j}\right)\right]\left(\bar{g}_{h l} \bar{g}_{i k}-\bar{g}_{h k} \bar{g}_{i l}\right)=K\left[\pi_{h}\left(\bar{g}_{i l} \varphi_{j k}-\bar{g}_{i k} \varphi_{j l}+\varphi_{i l} \bar{g}_{j k}-\varphi_{i k} \bar{g}_{j l}\right)+\right.} \\
& \left.\pi_{i}\left(\bar{g}_{j l} \varphi_{h k}-\bar{g}_{j k} \varphi_{h l}+\varphi_{j l} \bar{g}_{h k}-\varphi_{j k} \bar{g}_{h l}\right)+\pi_{j}\left(\bar{g}_{h l} \varphi_{i k}-\bar{g}_{h k} \varphi_{i l}+\varphi_{h l} \bar{g}_{i k}-\varphi_{h k} \bar{g}_{i l}\right)\right] .
\end{aligned}
$$

Multiplying both sides of this expression by $\bar{g}^{j k}$, and contracting the indices $\mathrm{j}, \mathrm{k}$, then we obtain

$$
\begin{aligned}
& (n-2)\left\{\left[\nabla_{h} K-K\left(\psi_{h}-2 \sigma_{h}\right)\right] \bar{g}_{i l}-\left[\nabla_{i} K-K\left(\psi_{i}-2 \sigma_{i}\right)\right] \bar{g}_{h l}\right\} \\
= & (n-3)\left(\pi_{h} \varphi_{i l}-\pi_{i} \varphi_{h l}\right)+\bar{g}_{i l}\left(\pi_{h} \varphi_{p}^{p}-\pi_{p} \varphi_{h}^{p}\right)-\bar{g}_{h l}\left(\pi_{i} \varphi_{p}^{p}-\pi_{p} \varphi_{i}^{p}\right) .
\end{aligned}
$$

And multiplying both sides of this expression again by $\bar{g}^{i l}$, and contracting the indices $\mathrm{i}, 1$, then we obtain

$$
(n-1)(n-2)\left[\nabla_{h} K-K\left(\psi_{h}-2 \sigma_{h}\right)\right]=2 K(n-2)\left(\pi_{h} \varphi_{p}^{p}-\pi_{p} \varphi_{h}^{p}\right) .
$$

From this expression we obtain

$$
\nabla_{h} K=K\left[\psi_{h}-2 \sigma_{h}+\frac{2}{n-1}\left(\pi_{h} \varphi_{p}^{p}-\pi_{p} \varphi_{h}^{p}\right)\right] .
$$

Consequently, for $n \geq 3, K=$ const, if and only if

$$
\psi_{h}-2 \sigma_{h}+\frac{2}{n-1}\left(\pi_{h} \varphi_{p}^{p}-\pi_{p} \varphi_{h}^{p}\right)=0 .
$$

Remark 2.3. If $\psi_{h}=0$, then the expression (2.15) is

$$
\sigma_{h}=\frac{1}{n-1}\left(\pi_{h} \varphi_{p}^{p}-\pi_{p} \varphi_{h}^{p}\right)
$$

and if $\sigma_{h}=0$, then the expression (2.15) is

$$
\psi_{h}=\frac{-2}{n-1}\left(\pi_{h} \varphi_{p}^{p}-\pi_{p} \varphi_{h}^{p}\right)
$$

And if $\psi_{h}=\sigma_{h}=0$, then the expression is

$$
\pi_{h} \varphi_{p}^{p}=\pi_{p} \varphi_{h}^{p}
$$

From Theorem 2.3, it is easy to see that there holds the following Corollary for the quarter-symmetric metric connection $\stackrel{q}{\nabla}$

Corollary 2.1. A connected $n$-dimensional Riemannian manifold $(M, g)($ dim $M \geq 3)$ associated with a quartersymmetric metric connection $\stackrel{q}{\nabla}$ being isotropic is a constant curvature manifold.

If $\varphi(X)=f X$, then quarter-symmetric projective conformal connection $\nabla$ will be expressed as $\mathrm{D}$. In this case the expressions (2.5) and (2.6) are respectively

$$
\begin{gathered}
D_{k} \bar{g}_{i j}=-2\left(\psi_{k}-\sigma_{k}\right) \bar{g}_{i j}-\psi_{i} \bar{g}_{j k}-\psi_{j} \bar{g}_{i k}, \quad T_{i j}^{k}=f\left(\pi_{j} \delta_{i}^{k}-\pi_{i} \delta_{j}^{k}\right), \\
\quad \Gamma_{i j}^{k}=\left\{\begin{array}{l}
k \\
i j
\end{array}\right\}+\left(\psi_{i}-\sigma_{i}\right) \delta_{j}^{k}+\left(\psi_{j}-\sigma_{j}+f \pi_{j}\right) \delta_{i}^{k}+\bar{g}_{i j}\left(\sigma^{k}-f \pi^{k}\right) .
\end{gathered}
$$

And the curvature tensor of the connection $\mathrm{D}$ is

$$
\stackrel{D}{R_{i j k}^{l}}=\bar{K}_{i j k}^{l}+\delta_{j}^{l} \alpha_{i k}-\delta_{i}^{l} \alpha_{j k}+\bar{g}_{j k} \beta_{i}^{l}-\bar{g}_{i k} \beta_{j}^{l}+\delta_{k}^{l} \psi_{i j},
$$


where

$$
\begin{aligned}
\alpha_{i k} & =\stackrel{0}{\nabla}_{i}\left(\psi_{k}-\sigma_{k}+f \pi_{k}\right)-\left(\psi_{i}-\sigma_{i}+f \pi_{i}\right)\left(\psi_{k}-\sigma_{k}+f \pi_{k}\right)-\bar{g}_{i k}\left(\psi_{p}-\sigma_{p}+f \pi_{p}\right)\left(\sigma^{p}-f \pi^{p}\right), \\
\beta_{i k} & =\stackrel{0}{\nabla_{i}}\left(\sigma_{k}-f \pi_{k}\right)+\left(\sigma_{i}-f \pi_{i}\right)\left(\sigma_{k}-f \pi_{k}\right) .
\end{aligned}
$$

A dual connection $\stackrel{*}{D}$ of the quarter-symmetric projective conformal connection D satisfies the relation

$$
\stackrel{*}{D_{k}} \bar{g}_{i j}=2\left(\psi_{k}-\sigma_{k}\right) \bar{g}_{i j}+\psi_{i} \bar{g}_{j k}+\psi_{j} \bar{g}_{i k}, \stackrel{*}{T_{i j}^{k}}=\left(\psi_{j}+f \pi_{j}-2 \sigma_{j}\right) \delta_{i}^{k}-\left(\psi_{i}+f \pi_{i}-2 \sigma_{i}\right) \delta_{j}^{k} .
$$

And from (2.19), its coefficient is

$$
\stackrel{\Gamma^{*}}{\Gamma_{i j}^{k}}=\left\{{ }_{i j}^{k}\right\}-\left(\psi_{i}-\sigma_{i}\right) \delta_{j}^{k}-\left(\sigma_{j}-f \pi_{j}\right) \delta_{i}^{k}-\bar{g}_{i j}\left(\psi^{k}-\sigma^{k}+f \pi^{k}\right) .
$$

And its curvature tensor is

$$
\stackrel{D *}{R_{i j k}^{l}}=\bar{K}_{i j k}^{l}+\delta_{i}^{l} \beta_{i k}-\delta_{j}^{l} \beta_{i k}+\bar{g}_{i k} \alpha_{j}^{l}-\bar{g}_{j k} \alpha_{i}^{l}-\delta_{k}^{l} \psi_{i j} .
$$

Theorem 2.4. If a Riemannian metric admits a quarter-symmetric projective conformal connection $D$ with a constant curvature on a Riemannian manifold $(M, g)($ dim $\geq 3)$, then the Riemannian metric is conformally flat.

Proof. Adding the expressions (2.20) and (2.21), and setting $\gamma_{i k}=\alpha_{i k}-\beta_{i k}$, we obtain

$$
\begin{gathered}
D \\
R_{i j k}^{l}+R_{i j k}^{l *}
\end{gathered}=2 \bar{K}_{i j k}^{l}+\delta_{j}^{l} \gamma_{i k}-\delta_{i}^{l} \gamma_{j k}+\bar{g}_{i k} \gamma_{j}^{l}-\bar{g}_{j k} \gamma_{i}^{l},
$$

Contracting the indices $i$ and 1 of (2.22), then we obtain

$$
\stackrel{D}{R_{j k}}+\stackrel{D *}{R_{j k}}=2 \bar{K}_{j k}-(n-2) \gamma_{j k}-\bar{g}_{j k} \gamma_{i}^{i},
$$

Multiplying both sides of (2.23) by $\bar{g}^{j k}$, we obtain

$$
\stackrel{D}{R}+\stackrel{D *}{R}=2 \bar{K}-2(n-1) \gamma_{i}^{i},
$$

From this expression

$$
\gamma_{i}^{i}=\frac{1}{2(n-1)}[2 \bar{K}-(\stackrel{D}{R}+\stackrel{D *}{R})],
$$

Using this expression, from (2.23), we obtain

$$
\left.\gamma_{j k}=\frac{1}{(n-2)}\left\{2 \bar{K}_{j k}-\left(\stackrel{D}{R_{j k}}+\stackrel{D *}{R_{j k}}\right)-\frac{\bar{g}_{j k}}{2(n-1)}[2 \bar{K}-\stackrel{D}{R}+\stackrel{D *}{R})\right]\right\},
$$

Substituting this expression into (2.22) and putting

$$
\begin{gathered}
\stackrel{D}{C_{i j k}^{l}}=\stackrel{D}{R_{i j k}^{l}}-\frac{1}{(n-2)}\left(\delta_{i}^{l} R_{j k}^{D}-\delta_{j}^{l} R_{i k}^{D}+\bar{g}_{j k} R_{i}^{l}-\bar{g}_{i k} R_{j}^{l}\right)-\frac{\stackrel{D}{R}}{(n-1)(n-2)}\left(\delta_{j}^{l} \bar{g}_{i k}-\delta_{i}^{l} \bar{g}_{j k}\right), \\
\stackrel{D *}{C_{i j k}^{l}}=\stackrel{R_{i j k}^{l}}{D^{*}}-\frac{1}{(n-2)}\left(\delta_{i}^{l} R_{j k}^{D *}-\delta_{j}^{l} R_{i k}^{D *}+\bar{g}_{j k} R_{i}^{l *}-\bar{g}_{i k} R_{j}^{l}\right)-\frac{D^{*}}{(n-1)(n-2)}\left(\delta_{j}^{l} \bar{g}_{i k}-\delta_{i}^{l} \bar{g}_{j k}\right), \\
\stackrel{0}{\bar{C}_{i j k}^{l}}=\bar{K}_{i j k}^{l}-\frac{1}{(n-2)}\left(\delta_{i}^{l} \bar{K}_{j k}-\delta_{j}^{l} \bar{K}_{i k}+\bar{g}_{j k} \bar{K}_{i}^{l}-\bar{g}_{i k} \bar{K}_{j}^{l}\right)-\frac{\bar{K}}{(n-1)(n-2)}\left(\delta_{j}^{l} \bar{g}_{i k}-\delta_{i}^{l} \bar{g}_{j k}\right),
\end{gathered}
$$

Then by a direct computation we obtain

$$
\stackrel{D}{C_{i j k}^{l}}+\stackrel{D *}{C_{i j k}^{l}}=2 \stackrel{0}{{ }^{l}}
$$

From $R_{i j k}^{l}=K\left(\delta_{j}^{l} \bar{g}_{i k}-\delta_{i}^{l} \bar{g}_{j k}\right)$, we have $C_{i j k}^{D}=\stackrel{D *}{C_{i j k}^{l}}=0$. Hence by (2.24), ${\stackrel{0}{C_{i j k}^{l}}}^{l}=0$. Using ${\overline{C_{i j k}^{l}}}^{0}=C_{i j k}^{l}$, then $C_{i j k}^{l}=0$, where $C_{i j k}^{l}=0$ is a Weyl conformal curvature tensor of a Riemannian metric $g_{i j}$. Hence if $n \geq 3$, then the Riemannian metric is conformal flat. 
Remark 2.4. The $C_{i j k}^{l}, C_{i j k}^{l *}$ and $\bar{C}_{i j k}^{l}$ are Weyl conformal curvature tensor of $\bar{g}_{i j}$ of $D, \stackrel{*}{D}$ and $\stackrel{0}{\nabla}$, respectively.

Theorem 2.5. The quarter-symmetric projective conformal connection $D$ is conjugate symmetric in a Riemannian manifold if and only if the corresponding Ricci curvature tensors are equal.

Proof. From the expressions (2.20) and (2.21), we get

$$
\stackrel{D *}{R_{i j k}^{l}}=\stackrel{D}{l} R_{i j k}^{l}+\delta_{i}^{l} \rho_{j k}-\delta_{j}^{l} \rho_{i k}+\bar{g}_{i k} \rho_{j}^{l}-\bar{g}_{j k} \rho_{i}^{l}-2 \delta_{k}^{l} \psi_{i j},
$$

where $\rho_{i k}=\alpha_{i k}+\beta_{i k}$. Contracting the indices $\mathrm{i}$ and 1 we obtain

$$
\stackrel{D *}{R_{j k}}=\stackrel{D}{R_{j k}}+n \rho_{j k}-\bar{g}_{j k} \rho_{i}^{i}-2 \psi_{k j} .
$$

Alternating the indices $\mathrm{j}$ and $\mathrm{k}$ of this expression, using $\rho_{j k}-\rho_{k j}=\psi_{j k}$ we obtain,

$$
\stackrel{D *}{R_{j k}}-\stackrel{D *}{R_{k j}}=\stackrel{D}{R_{j k}}-\stackrel{D}{R_{k j}}+(n+4) \psi_{j k} .
$$

From this relation we find

$$
\psi_{j k}=\frac{1}{n+4}\left[\left(\stackrel{D}{R}_{j k}^{*}-\stackrel{D *}{R_{k j}}\right)-\left(\stackrel{D}{R_{j k}}-\stackrel{D}{R_{k j}}\right)\right]
$$

Using this expression, from (2.26)

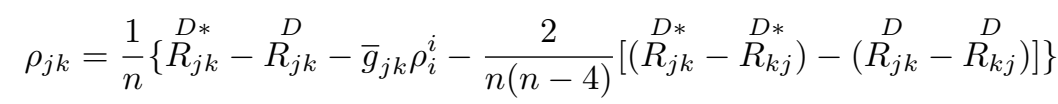

Substituting the above two expressions into (2.25)

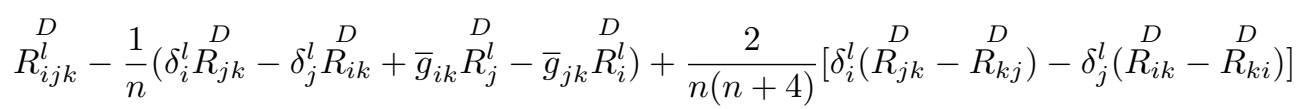

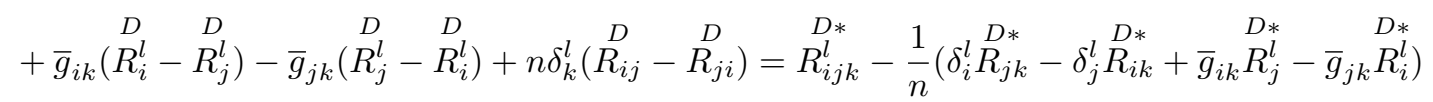

$$
\begin{aligned}
& +\frac{2}{n(n+4)}\left[\delta_{i}^{l}\left(\stackrel{D *}{R_{j k}}-\stackrel{D *}{R_{k j}}\right)-\delta_{j}^{l}\left(\stackrel{D *}{R_{i k}}-\stackrel{D_{*}^{*}}{R_{k i}}\right)\right]+\bar{g}_{i k}\left(\stackrel{D *}{R_{i}^{l}} \stackrel{D^{*}}{R_{j}^{l}}\right)-\bar{g}_{j k}\left(R_{j}^{l}-\stackrel{D *}{R_{i}^{l}}\right)+n \delta_{k}^{l}\left(\stackrel{D *}{R_{i j}}-\stackrel{D^{*}}{R_{j i}}\right) .
\end{aligned}
$$

From this expression we arrive at that $R_{i j k}^{l}=\stackrel{D *}{R_{i j k}^{l}}$ if and only if $\stackrel{D *}{R_{j k}}=\stackrel{D}{R_{j k}}$.

Now we will study the Schur's theorem of the quarter-symmetric projective conformal connection D. From the Theorem 2.3, the quarter-symmetric projective conformal connection D satisfies the Schur's theorem if and only if, from (2.15),

$$
\psi_{h}-2 \sigma_{h}+2 f \pi_{h}=0
$$

Hence, from (2.18) and (2.19) in a connected Riemannian manifold $(M, g)(\operatorname{dim} \geq 3)$, the quarter-symmetric projective conformal connection D satisfying the Schur's theorem satisfies

$$
D_{k} \bar{g}_{i j}=-2\left(\sigma_{k}-2 f \pi_{k}\right) \bar{g}_{i j}-2\left(\sigma_{i}-f \pi_{i}\right) \bar{g}_{j k}-2\left(\sigma_{j}-f \pi_{j}\right) \bar{g}_{i k}, T_{i j}^{k}=f\left(\pi_{j} \delta_{i}^{k}-\pi_{i} \delta_{j}^{k}\right) .
$$

And its connection coefficient is

$$
\stackrel{D}{\Gamma_{i j}^{k}}=\left\{\begin{array}{l}
k \\
i j
\end{array}\right\}+\left(\sigma_{i}-2 f \pi_{i}\right) \delta_{j}^{k}+\left(\sigma_{j}-2 f \pi_{j}\right) \delta_{i}^{k}+\bar{g}_{i j}\left(\sigma^{k}-f \pi^{k}\right) .
$$

From this fact, the quarter-symmetric projective conformal connection D satisfying the Schur's theorem is as follows.

1. $\sigma_{i}=0\left(D=\stackrel{p}{D}, \psi_{h}=-2 f \pi_{h}\right)$,

$$
\begin{aligned}
& D_{k}^{p} g_{i j}=2 f\left(2 \pi_{k} g_{i j}+\pi_{i} g_{j k}+\pi_{j} g_{i k}\right), T_{i j}^{k}=f\left(\pi_{j} \delta_{i}^{k}-\pi_{i} \delta_{j}^{k}\right), \\
& \stackrel{D p}{\Gamma_{i j}^{k}}=\left\{\begin{array}{l}
k \\
i j
\end{array}\right\}-f\left(2 \pi_{i} \delta_{j}^{k}+\pi_{j} \delta_{i}^{k}+g_{i j} \pi^{k}\right) .
\end{aligned}
$$


2. $\psi_{i}=0\left(D=\stackrel{c}{D}, \sigma_{h}=f \pi_{h}\right)$,

$$
\begin{aligned}
& {\stackrel{c}{{ }_{k}} \bar{g}_{i j}}_{i j}=2 f \pi_{k} \bar{g}_{i j}, T_{i j}^{k}=f\left(\pi_{j} \delta_{i}^{k}-\pi_{i} \delta_{j}^{k}\right), \\
& \Gamma_{i j}^{k}=\overline{\left\{{ }_{i j}^{k}\right\}}-f \pi_{i} \delta_{j}^{k} .
\end{aligned}
$$

3. $\psi_{i}=\sigma_{i}$

$$
\begin{aligned}
& D_{k} \bar{g}_{i j}=-\psi_{i} \bar{g}_{j k}-\psi_{j} \bar{g}_{i k}, T_{i j}^{k}=f\left(\pi_{j} \delta_{i}^{k}-\pi_{i} \delta_{j}^{k}\right), \\
& \stackrel{D}{\Gamma_{i j}^{k}}=\overline{\left\{{ }_{i j}^{k}\right\}}+f \pi_{j} \delta_{i}^{k}+\bar{g}_{i j}\left(\psi^{k}-f \pi^{k}\right) .
\end{aligned}
$$

\section{Acknowledgments}

The third and fourth author would like to thank Professors H. Li, Z. Hu, Z. Sun, Z. Li and H. Song for their encouragement and help! The authors wish to thank the referees for their valuable comments and suggestions.

\section{References}

[1] De, U. C. and Biswas, S. C., Quarter-symmetric metric connection in an SP-Sasakian manifold, Commun. Fac. Sci. Univ. Ank. Series Al.. 46(1997), 49-56.

[2] De, U. C. and Biswas, S. C., On a type of semi-symmetric metric connection on a Riemannian manifold, Publ. Inst. Math.(Beograd)(N. S. ). 61(1997), no.75, 90-96.

[3] De, U. C. and Kamilya, D., Hypersurfaces of Kenmotsu manifolds endowed with a quarter-symmetric non-metric connection , Kuwait J. Sci. Eng.. 39( 2012), no.1A, 43-56.

[4] De, U. C. and Mondal, A. K., Quarter-symmetric metric connection on 3-dimensional quasi-Sasakian manifolds, SUT Journal of Mathematics. 46(2010), no.1, 35-52.

[5] De, U. C. and Sengupta, J., On a type of semi-symmetric non-metric connection on a Riemannian manifold, Bull. Cal. Math. Soc.. 92(2000),375-884.

[6] Fridman, A. and Schouten, J. A., Uber die Geometric der halb-symmerischen Ubertrngungen, Math.Zeitschift. 21(1924), 211-233.

[7] Han, Y. L., Ho, Tal Yun. and Zhao, P. B., Some invariants of quarter-symmetric metric connections under the projective transformation, Filomat. 27(2013), no.4, 679-691.

[8] Han, Y. L., Ho, Tal Yun. and Zhao, P. B., A Schur's lemma based on a semi-symmetric non-metric connection, International Journal of Geometry. 5(2016), no.1, 47-53.

[9] Han, Y. L. and Zhao, P. B., A Class of nearly sub-Weyl and sub-Lyra Manifolds, accepted in International Electronic Journal of Geometry. (2016).

[10] Hayden, H. A., Subspace of space with torsion, Proc. of London Math. Soc.. 24 (1932), 27-50.

[11] Stepanova, E. S., Dual symmetric statistical manifold. J. of Mathematical Sciences. 147(2007), no.1, 6507-6509.

[12] Yano, K., On semi-symmetric metric connection, Rev. Roun. Math. Purest. Appl.. 15(1971), 1579-1586.

[13] Yano, K. and Imai, J., Quarter-symmetric metric connection and their curvature tensors, Tensor. N. S.. 38(1982), 13-18.

[14] Ho, Tal Yun., On a semi-symmetric non-metric connection satisfying the Schur's theorem on a Riemannian manifold, arXiv:1212,4748v1.

[15] Ho, Tal Yun., On the projective semi-symmetric connection and the conformal semi-symmetric connection on the Riemannian manifold, J. of Kim Il Sung University (Natural Science). 2(2013), no.2, 3-10.

[16] Ho, Tal Yun., An, Jae Hyon and An, Chang Gil., Some properties of mutual connection of semi-symmetric metric connection and its dual connection in a Riemannian manifold, Acta Scientiarum Naturalium Universtatis Nankaiensts. 46(2013), no.4, 1-8.

[17] Ho, Tal Yun., Jen, Cholyong and Jin, Guangzhi., A semi-symmetric projective conformal connection satisfying the Schur's theorem on a Riemannian manifold, J. of Yanbian University (Natural Science). 40(2014), no.4, 290-294.

[18] Zhao, P. B., Some properties of projective semi-symmetric connections, International Mathematical Forum. 3(2008), no. 7, 341-347.

[19] Zhao, P. B. and Song, H. Z., An invariant of the projective semi-symmetric connection, Chinese Quarterly J. of Math.. 17(2001), no. 4, 48-52.

[20] Zhao, P. B., Song, H. Z. and Yang, X. P., Some invariant properties of the semi-symmetric metric recurrent connections and curvature tensor expressions, Chinese Quarterly J. of Math.. 19(2004), no. 4, 355-361.

\section{Affiliations}

WANXIAO TANG

AdDress: Nanjing University of Science and Technology, Dept. of Applied Mathematics, 210094, Nanjing, P. R. China. 
E-MAIL: wanxiaotang92@163.com

ORCID ID : orcid.org/0000-0003-1343-4994

TAL YUN HO

AdDress: Kim Tl Sung University, Dept. of Mathematics, Pyongyang, Democratic Peoples Republic of Korea. E-MAIL: hochong@163.com

ORCID ID : orcid.org/0000-0002-6750-8736

FENGYUN FU

AdDrEss: Guangdong University of Finance and Economics, Dept. of Mathematics and Statistics, 510320, Guangzhou, P. R. China.

E-MAIL: fengyunfu@aliyun.com

ORCID ID : orcid.org/0000-0002-3417-9526

Peibiao Zhao

AdDress: Nanjing University of Science and Technology, Dept. of Applied Mathematics, 210094, Nanjing, P. R. China.

E-MAIL: pbzhao@njust.edu.cn

ORCID ID : orcid.org/0000-0002-0262-5544 